\section{Ropeginterferon alfa-2b versus standard therapy for polycythaemia vera (PROUD-PV and CONTINUATION-PV): a randomised, non-inferiority, phase 3 trial and its extension study}

\author{
Heinz Gisslinger, Christoph Klade, Pencho Georgiev, Dorota Krochmalczyk, Liana Gercheva-Kyuchukova, Miklos Egyed, Viktor Rossiev, Petr Dulicek, \\ Arpad Illes, Halyna Pylypenko, Lylia Sivcheva, Jiri Mayer, Vera Yablokova, Kurt Krejcy, Barbara Grohmann-Izay, Hans C Hasselbalch, \\ Robert Kralovics, Jean-Jacques Kiladjian, for the PROUD-PV Study Group*
}

\section{Summary}

Background The PROUD-PV and CONTINUATION-PV trials aimed to compare the novel monopegylated interferon ropeginterferon alfa-2b with hydroxyurea, the standard therapy for patients with polycythaemia vera, over 3 years of treatment.

Methods PROUD-PV and its extension study, CONTINUATION-PV, were phase 3, randomised, controlled, openlabel, trials done in 48 clinics in Europe. Patients were eligible if 18 years or older with early stage polycythaemia vera (no history of cytoreductive treatment or less than 3 years of previous hydroxyurea treatment) diagnosed by WHO's 2008 criteria. Patients were randomly assigned 1:1 to ropeginterferon alfa-2b (subcutaneously every 2 weeks, starting at $100 \mu \mathrm{g}$ ) or hydroxyurea (orally starting at $500 \mathrm{mg} / \mathrm{day}$ ). After 1 year, patients could opt to enter the extension part of the trial, CONTINUATION-PV. The primary endpoint in PROUD-PV was non-inferiority of ropeginterferon alfa-2b versus hydroxyurea regarding complete haematological response with normal spleen size (longitudinal diameter of $\leq 12 \mathrm{~cm}$ for women and $\leq 13 \mathrm{~cm}$ for men) at 12 months; in CONTINUATION-PV, the coprimary endpoints were complete haematological response with normalisation of spleen size and with improved disease burden (ie, splenomegaly, microvascular disturbances, pruritus, and headache). We present the final results of PROUD-PV and an interim analysis at 36 months of the CONTINUATION-PV study (per statistical analysis plan). Analyses for safety and efficacy were per-protocol. The trials were registered on EudraCT, 2012-005259-18 (PROUD-PV) and 2014-001357-17 (CONTINUATION-PV, which is ongoing).

Findings Patients were recruited from Sept 17, 2013 to March 13, 2015 with 306 enrolled. 257 patients were randomly assigned, 127 were treated in each group (three patients withdrew consent in the hydroxyurea group), and 171 rolled over to the CONTINUATION-PV trial. Median follow-up was 182.1 weeks (IQR 166.3-201.7) in the ropeginterferon alfa-2b and 164. 5 weeks (144.4-169.3) in the standard therapy group. In PROUD-PV, $26(21 \%)$ of 122 patients in the ropeginterferon alfa-2b group and $34(28 \%)$ of 123 patients in the standard therapy group met the composite primary endpoint of complete haematological response with normal spleen size. In CONTINUATION-PV, complete haematological response with improved disease burden was met in 50 (53\%) of 95 patients in the ropeginterferon alfa-2b group versus 28 (38\%) of 74 patients in the hydroxyurea group, $\mathrm{p}=0.044$ at 36 months. Complete haematological response without the spleen criterion in the ropeginterferon alfa- $2 b$ group versus standard therapy group were: $53(43 \%)$ of 123 patients versus 57 (46\%) of 125 patients, p=0.63 at 12 months (PROUD-PV), and 67 (71\%) of 95 patients versus 38 (51\%) of 74 patients, p=0.012 at 36 months (CONTINUATION-PV). The most frequently reported grade 3 and grade 4 treatment-related adverse events were increased $\gamma$-glutamyltransferase (seven [6\%] of 127 patients) and increased alanine aminotransferase (four [3\%] of 127 patients) in the ropeginterferon alfa-2b group, and leucopenia (six [5\%] of 127 patients) and thrombocytopenia (five [4\%] of 127 patients) in the standard therapy group. Treatment-related serious adverse events occurred in three (2\%) of 127 patients in the ropeginterferon alfa- $2 \mathrm{~b}$ group and five $(4 \%)$ of 127 patients in the hydroxyurea group. One treatment-related death was reported in the standard therapy group (acute leukaemia).

Interpretation In patients with early polycythaemia vera, who predominantly presented without splenomegaly, ropeginterferon alfa-2b was effective in inducing haematological responses; non-inferiority to hydroxyurea regarding haematological response and normal spleen size was not shown at 12 months. However, response to ropeginterferon alfa- $2 \mathrm{~b}$ continued to increase over time with improved responses compared with hydroxyurea at 36 months. Considering the high and durable haematological and molecular responses and its good tolerability, ropeginterferon alfa-2b offers a valuable and safe long-term treatment option with features distinct from hydroxyurea.
Lancet Haematol 2020 7: e196-208

Published Online January 31,2020 https://doi.org/10.1016/ \$2352-3026(19)30236-4 This online publication has been corrected. The corrected version first appeared at thelancet.com/haematology on Feb 25, 2020

See Comment page e178 ${ }^{*}$ A complete list of the PROUD-PV Study Group investigators is in the appendix

Department of Internal Medicine I, Division of Haematology and Blood Coagulation (H Gisslinger MD) and Department of Laboratory Medicine (R Kralovics PhD), Medical University Vienna, Vienna, Austria; AOP Orphan Pharmaceuticals AG, Vienna, Austria (C Klade PhD, K Krejcy MD

B Grohmann-Izay MD); University Multiprofile Hospital for Active Treatment "Sveti Georgi", Clinic of Haematology, Medical University of Plovdiv, Plovdiv Bulgaria (P Georgiev MD); Teaching Unit of the Haematology Department, University Hospital in Krakow, Krakow, Poland (D Krochmalczyk MD); Multiprofile Hospital for Active Treatment "Sveta Marina", Clinical Haematology Clinic, Varna, Bulgaria (L Gercheva-Kyuchukova MD); Department of Interna Medicine II, Kaposi MorCounty Teaching Hospital, Kaposvar, Hungary (M Egyed MD); Samara Kalinin Regional Clinical Hospital, Samara, Russia (V Rossiev MD); Department of Clinical Haematology, University Hospital Hradec 
Kralove, Hradec Kralove, Czech Republic (P Dulicek MD); Department of Haematology, Faculty of Medicine, University of Debrecen, Debrecen, Hungary (A Illes MD); Department of Haematology, Regional Treatment and Diagnostics Haematology Centre, Cherkasy Regional Oncology Centre, Cherkasy,

Ukraine (H Pylypenko MD); Multiprofile Hospital for Active Treatment-HristoBotev, First Department of Internal Medicine, Vratsa, Bulgaria (L Sivcheva MD); Clinic of Internal MedicineHaematology and Oncology, University Hospital Brno, Brno,

Czech Republic (J Mayer MD); Yaroslavl Regional Clinical Hospital, Department of Haematology, Yaroslavl, Russia (VYablokova MD); Department of Haematology, Zealand University Hospital, Roskilde, University of Copenhagen,

Denmark (HC Hasselbalch MD); CeMM Research Center for Molecular Medicine of the Austrian Academy of Sciences, Vienna, Austria (R Kralovics); Université de Paris, CIC 1427, Inserm, F-75010, Paris, France (J-J Kiladjian MD); and Centre d'Investigations Cliniques, AP-HP, Hopital Saint-Louis,

F-75010, Paris, France (J-J Kiladjian)

Correspondence to: Dr Heinz Gisslinger, Department of Internal Medicine I, Division of Haematology and Blood Coagulation, Medical University Vienna, Vienna A-1090, Austria heinz.gisslinger@meduniwien.

\section{Funding AOP Orphan Pharmaceuticals AG.}

\section{Copyright (C) 2020 Elsevier Ltd. All rights reserved.}

\section{Introduction}

Polycythaemia vera, as a subgroup of the myeloproliferative neoplasms, is characterised by uncontrolled malignant proliferation of haematopoietic cells leading to increased concentrations of red cells, often accompanied by elevated leucocyte and platelet counts. ${ }^{1-3}$ The oncogenic pathway in polycythaemia vera is driven by the Val617Phe mutation in the JAK2 gene present in about $95 \%$ of patients, which results in constitutive kinase activity promoting both haematopoietic cell proliferation and a proinflammatory state. ${ }^{3-7}$ The main clinical manifestations include pruritus, fatigue, and microcirculatory disturbances. Patients with polycythaemia vera are predisposed to thrombosis and disease progression to myelofibrosis or secondary leukaemia, which results in poor prognosis. ${ }^{1,3}$ Besides phlebotomy and low dose aspirin, cytoreductive therapy improves symptoms and might prevent thromboembolic events. $^{1,2}$

Among cytoreductive agents used to treat polycythaemia vera, interferon alfa (IFN $\alpha$ ) has been consistently reported to have a disease-modifying capacity by selectively decreasing the malignant stem cell pool and inducing durable molecular remissions in some patients..$^{8-16}$ These properties suggest that IFN $\alpha$ might be of greatest benefit when used early in the course of the disease to inhibit the clonal evolution that underlies disease progression. ${ }^{13}$ Clinical and haematological efficacy of IFN $\alpha$-based therapy in myeloproliferative neoplasms has been reported since the 1980s; however, toxicity and the need for frequent parenteral application of

Research in context

Evidence before this study

Interferon alfa has been used for more than 30 years for treatment of myeloproliferative neoplasms. However, conventional interferons are generally considered to be associated with a high frequency of adverse events and treatment withdrawals. The introduction of pegylated interferon compounds improved tolerability and proportion of withdrawals. Since the discovery of Val617Phe in the JAK2 gene as a driver mutation, and thereby the opportunity to monitor molecular remissions in myeloproliferative neoplasms, pegylated interferons have been increasingly used off-label as a non-leukaemogenic alternative to hydroxyurea, the standard, first-line cytoreductive treatment for polycythaemia vera. Although this practice is supported by some international guidelines based on the results of independent, single arm, phase 2 clinical trials, this process has remained a point of controversy because no randomised, phase 3 , clinical trial comparing pegylated interferon alfa with hydroxyurea was previously available as evidence-based guidance for clinical conventional formulations led to high proportions of patients discontinuing treatment., ${ }^{4,911,17-23}$

Ropeginterferon alfa- $2 \mathrm{~b}$ is a monopegylated IFNa developed for treating myeloproliferative neoplasms. In contrast to other pegylated IFN $\alpha$ compounds, ropeginterferon alfa- $2 \mathrm{~b}$ consists of a single positional isomer resulting in an extended elimination half-life, enabling less frequent dosing (every other week, or monthly during maintenance therapy) and improved tolerability, supporting long-term patient compliance. ${ }^{19}$

After more than 30 years of clinical use of IFN $\alpha$ in myeloproliferative neoplasms, this phase 3 clinical programme in an early polycythaemia vera population (comprising the PROUD-PV study for the first year of treatment and its extension study CONTINUATION-PV for a further 2 years) provides, to our knowledge, the first, randomised, controlled, head-to-head comparison of an IFN $\alpha$ with hydroxyurea, the current standard firstline cytoreductive treatment.

\section{Methods \\ Study design and participants}

PROUD-PV and its extension study CONTINUATION-PV were multicentre, open-label, active-controlled, phase 3 trials done in 48 clinics in Europe (appendix pp 31-33). In PROUD-PV, patients were randomly assigned to receive either ropeginterferon alfa-2b or hydroxyurea for 12 months; cross-over was not permitted. The primary objective in PROUD-PV was originally to assess the superiority of ropeginterferon alfa-2b versus hydroxyurea practice. We searched PubMed for publications of clinical trials in English before Oct 11, 2019, using the terms "interferon", "polycythaemia vera", and "randomised controlled trial", which showed no published randomised, controlled, phase 3 trials comparing interferon alfa with hydroxyurea in patients with polycythaemia vera.

\section{Added value of this study}

This research provides evidence in a randomised setting that ropeginterferon alfa- $2 \mathrm{~b}$ is more effective in achieving durable haematological and molecular remissions than hydroxyurea, the standard cytoreductive therapy, in patients with polycythaemia vera. In addition, the data show that this type of interferon treatment is well tolerated during long-term application.

\section{Implications of all the available evidence}

Taken together with pre-existing evidence, these findings suggest that ropeginterferon alfa- $2 \mathrm{~b}$ can be considered as first-line cytoreductive therapy instead of hydroxyurea and should therefore change treatment algorithms for polycythaemia vera. 
regarding disease response. Before database lock or sponsor unblinding, newly published data indicated that the haematological response among patients treated with hydroxyurea might exceed the $15 \%$ assumed in PROUD-PV, which was estimated based on earlier literature. ${ }^{24,25}$ Therefore, in a protocol amendment dated June 15, 2016, the study objective in PROUD-PV was revised to the assessment of non-inferiority of ropeginterferon alfa- $2 \mathrm{~b}$ versus hydroxyurea regarding disease response.

Findings from both the completed PROUD-PV study and the ongoing study CONTINUATION-PV are presented; the interim analysis of CONTINUATION-PV data occurred when all patients had reached month 36 or discontinued the trial. The extension study is planned to last for 5 years (ie, 6 years in total for PROUD-PV and CONTINUATION-PV).

The main eligibility criteria were age ( $\geq 18$ years) and a diagnosis with polycythaemia vera, according to WHO's 2008 criteria, ${ }^{26}$ including a JAK2 Val617Phe mutation. Patients with no history of cytoreduction required a documented need of cytoreductive therapy. Patients previously treated with hydroxyurea for less than 3 years were eligible if they could potentially benefit from participation in the trial, having no complete response, resistance or intolerance to hydroxyurea according to modified European LeukemiaNet criteria. Patients with previous cytoreductive treatment other than hydroxyurea were ineligible. Major comorbidities that could be exacerbated by IFN $\alpha$ treatment were exclusion criteria; a complete list of inclusion and exclusion criteria is provided in the appendix pp 8-10. Stable haematocrit of less than $45 \%$ was required for randomisation; if the haematocrit exceeded $45 \%$ before randomisation, phlebotomy was done.

Only patients who completed the PROUD-PV trial were eligible for the extension study. To be included in this part, patients had to have normalisation of at least two of three main blood parameters (haematocrit, platelets, and white blood cells), or a reduction of more than $35 \%$ in at least two of three main blood parameters from baseline, or normalisation of spleen size, or clinically confirmed benefit from treatment with ropeginterferon alfa- $2 b$ (ie, normalisation of disease-related micro-vasculatory symptoms, substantial decrease of JAK2 Val617Phe allelic burden).

Patients provided written informed consent. The trials were done in accordance with the Declaration of Helsinki and International Conference on Harmonization Good Clinical Practice Guidelines. Protocols were approved by the institutional review board or independent ethics committees at each site. An independent data monitoring committee oversaw the studies and could recommend their termination based on a risk-benefit assessment.

\section{Randomisation and masking}

Patients were randomly assigned 1:1 to ropeginterferon alfa-2b or hydroxyurea. Randomisation was block-stratified by eight strata (previous hydroxyurea treatment [yes or no], age at screening [ $\leq 60$ years or $>60$ years], and history of thromboembolic events [yes or no]). Patients were defined as enrolled on providing the investigator or delegate with their written informed consent. Treatment assignment was done at the sites using an electronic case report form system (Merge eClinical OS). The randomisation list was generated by the statistician using Statistical Analysis System (SAS) version 9.3. PROUD-PV was not blinded; however, the sponsor had no access to cumulative data before database lock.

\section{Procedures}

In the 12 month, PROUD-PV study, ropeginterferon alfa-2b was administered subcutaneously every 2 weeks at a starting dose of $100 \mu \mathrm{g}$, or $50 \mu \mathrm{g}$ if the patient was transitioning from hydroxyurea. Hydroxyurea was administered orally starting at $500 \mathrm{mg}$ per day. Dosing was increased until the haematocrit was less than $45 \%$ without phlebotomy and normalised platelet and leucocyte counts (platelet count $<400 \times 10^{9} / \mathrm{L}$, and leucocyte count $<10 \times 10^{9} / \mathrm{L}$ ) were reached and maintained (defined by the investigator's discretion). Patients transitioning from pretrial hydroxyurea to ropeginterferon alfa-2b followed a predefined dosing scheme (appendix p 21). All patients received low-dose aspirin during the trial unless contraindicated.

Patients who completed PROUD-PV were eligible to roll over into the extension study CONTINUATION-PV, in which patients randomly assigned to ropeginterferon alfa-2b in PROUD-PV continued this treatment. Patients from the standard therapy group in PROUD-PV received best available treatment selected by the investigator, which could include hydroxyurea, conventional IFN $\alpha$ or pegylated IFNa (other than ropeginterferon alfa-2b), anagrelide, a JAK2 inhibitor, phosphorus-32, or busulfan.

After randomisation in PROUD-PV (baseline), patients attended efficacy assessment visits every 3 months; this schedule of assessments continued during CONTINUATION-PV and comprised haematocrit, platelet count, leucocyte count, erythrocyte count, requirement for phlebotomy, spleen size, quality of life (determined using the European Quality of Life, 5 dimensions, 3 levels [EQ-5D-3L] questionnaire, only the total score is available (derived from the five dimensions mobility, self-care, usual activities, pain or discomfort, and anxiety or depression); results were not analysed for each individual dimension of the global scale, and disease burden (ie, splenomegaly, microvascular disturbances, pruritus, and headache). In PROUD-PV, haematological tests for the primary endpoint were assessed centrally; local site laboratories were used in CONTINUATION-PV. Spleen size was determined by blinded central MRI or CT at month 12 for the assessment of the primary endpoint in PROUD-PV and at the local sites using ultrasound sonography at all other timepoint (every 3 months thereafter). Quantitative JAK2 Val617Phe allelic burden 
(JAK2V617F ipsogen JAK2 MutaQuant kit, QIAGEN $\mathrm{GmbH}$, Hilden, Germany) was assessed centrally every 6 months. Centrally reviewed bone marrow histology was mandatory to confirm polycythaemia vera at baseline if used as a major diagnostic criterion; additionally, optional bone marrow samples were collected at screening, at 12 months, and at the patient's final visit and assessed by an independent pathology board.

Throughout both studies, safety was evaluated at each patient visit for treatment administration, based on reported adverse events, urinalysis, haematology, and clinical chemistry according to Common Terminology Criteria for Adverse Events (CTCAE) 4.0. Additional safety assessments were done at intervals of 3 months, including a physical examination, evaluation of coagulation parameters, immunological parameters, Eastern Cooperative Oncology Group performance status, Hospital Anxiety and Depression Scale (HADS), and if clinically indicated, ocular examination and chest radiograph. Standard 12-lead electrocardiograms and echocardiograms were done at intervals of 3 months for the first year and at 6 months thereafter.

Dose interruption was permitted for grade 3 or higher toxicity and dose reduction for grade 2 toxicity. Treatment was discontinued in the case of unresolved treatmentrelated toxicity, HADS score of at least 11 (either subscale), suicidal ideation, or clinically significant depression, increased hepatic enzyme levels, autoimmune disease, ophthalmological disorder, or loss of efficacy of ropeginterferon alfa-2b.

\section{Outcomes}

The primary efficacy endpoint in PROUD-PV was a composite outcome comprising both complete haematological response (defined as haematocrit $<45 \%$ with no phlebotomy in the past 3 months, platelet count $<400 \times 10^{9} / \mathrm{L}$, and leucocyte count $<10 \times 10^{9} / \mathrm{L}$ ) and normal spleen size by imaging (longitudinal diameter of $\leq 12 \mathrm{~cm}$ for women and $\leq 13 \mathrm{~cm}$ for men) at month 12 .

The secondary endpoints were complete haematological response as an independent factor (without spleen size), change in JAK2 Val617Phe allelic burden, haematological parameters, and spleen size from baseline, time to disease response, disease response duration, phlebotomy need, change of disease-related symptoms (microvascular disturbances, pruritus, headache), change in quality of life (EQ-5D-3L) from baseline, and adverse events. Other secondary endpoints that were added to the Statistical Analysis Plan included molecular response: complete response is defined as a reduction of any molecular abnormality to undetectable levels; partial response is defined as a reduction in the JAK2 Val617Phe allele burden of at least $50 \%$ from baseline levels if the baseline value is less than $50 \%$, and a reduction of at least $25 \%$ from baseline if the baseline level is at least $50 \%{ }^{27}$ Patients that partially responded must have had a baseline allele burden more than $10 \%$.
The primary objective in CONTINUATION-PV was long-term assessment of efficacy and safety for ropeginterferon alfa-2b. The coprimary efficacy endpoints were the proportion of patients achieving a complete haematological response and normal spleen size (as defined in PROUD-PV) and a complete haematological response with improved disease burden (ie, resolution or clinical improvement of disease-related splenomegaly, microvascular disturbances, pruritus and headache, or a combination). The secondary endpoints were: change in JAK2V617F allelic burden, haematological parameters, and spleen size from baseline, time to disease response, maintenance of disease response, duration of response maintenance, progression-free time, phlebotomy need, change of disease related signs and disease-related symptoms, quality of life and adverse events. Other secondary endpoints that were added to the Statistical Analysis Plan included molecular response, defined as in PROUD-PV.

An overview of all endpoints is provided in the appendix p 22.

\section{Statistical methods}

The null hypothesis in PROUD-PV was inferiority of the response for ropeginterferon alfa-2b by at least $10 \cdot 5 \%$ of the response for hydroxyurea after 12 months. This hypothesis was tested against the alternative at a one-sided significance level of $2 \cdot 5 \%$ by estimating the two-sided $95 \%$ CI of the difference in response between the treatment groups and comparing the lower limit of the confidence interval to the non-inferiority margin. The noninferiority margin of $10.5 \%$ in PROUD-PV was derived by halving the assumed control effect of $25 \%$, after subtracting $4 \%$ to allow for random fluctuations in response due to the nature of the disease $([25 \%-4 \%] / 2=10 \cdot 5 \%)$. No formal hypothesis testing was done in the CONTINUATION-PV study.

The sample size of PROUD-PV was determined according to the anticipated size of treatment effect for the overall response at 12 months (at least $25 \%$ ). The assumed responses were $32 \%$ (ropeginterferon alfa- $2 \mathrm{~b}$ ) and $12 \%$ (standard therapy), after allowing for a 20\% drop-out rate (dropouts were considered non-responders). Thus, 252 patients (126 per group) were required to detect the difference in response rate between treatment groups at $1 \%$ (two-sided) significance level with $90 \%$ power, using a standard $\chi^{2}$ test. Considering divisibility by eight strata 128 patients per treatment group (256 in total) were planned to be enrolled. An interim analysis was planned with the possibility to reassess sample size but was deemed unnecessary by the independent Data Monitoring Committee. Following the change in the PROUD-PV study objective from superiority to non-inferiority of ropeginterferon alfa- $2 \mathrm{~b}$ versus hydroxyurea, and before sponsor unblinding, a blinded power simulation was done using the pooled sample sizes in each stratum and the defined non-inferiority margin of $10 \cdot 5 \%$. This 
calculation showed that a difference of 0.04 in responses between the treatment groups (in favour of the test drug) would provide statistical power of $81 \%$, which was considered to be sufficient and did not require an increase in sample size. No formal hypothesis was planned to be tested in the CONTINUATION-PV study; therefore, we did not do a power calculation or sample size calculation.

Primary analyses of efficacy in the PROUD-PV study were done using the Cochran-Mantel-Haenszel test with the defined stratification factors. Differences in the response between treatment groups and the 95\% CIs were calculated. Further, a logistic regression model was used to explore differences in response between hydroxyurea-exposed patients and patients with no previous history of hydroxyurea use. In the interim analysis of the CONTINUATION-PV study, comparisons of efficacy among the treatment groups were done using a $\log$ binomial regression model. The rate ratio (RR) of responders between treatment groups and the $95 \%$ CIs were calculated from estimates of regression coefficients. Two sensitivity analyses of the primary endpoint were done: complete haematological response (without the spleen criterion) and complete haematological response with improved disease burden where splenomegaly is defined as spleen size more than $17 \mathrm{~cm}$ (replacing the investigators assessment).

Efficacy analysis was done per protocol (patients analysed by treatment assigned; full analysis set). Safety analyses included all patients who received at least on dose of treatment. Additionally, a post-hoc analysis was done to determine the Pearson correlation coefficient between complete haematological response and JAK2 Val617Phe allelic burden per treatment group and assessment visit.

All analyses were done using SAS software, version 9.3 or higher (SAS Institute, Cary, NC, USA). The trials were registered on EudraCT, PROUD-PV: 2012-005259-18; CONTINUATION-PV: 2014-001357-17.

\section{Role of the funding source}

In collaboration with the investigators, the funder contributed to the study design, analysis, and interpretation of data. The investigators collected the clinical data. Contract research organisations monitored the trials and analysed the data. All authors had full access to all the raw data in the studies, reviewed the manuscript, and vouched for the accuracy and completeness of the data and analysis, and for the fidelity of the trials to the protocols. The corresponding author had final responsibility for the decision to submit for publication.

\section{Results}

In PROUD-PV we recruited 306 patients from Sept 17, 2013 to March 13, 2015. In PROUD-PV, of 257 patients randomly assigned, 127 were allocated and received ropeginterferon alfa- $2 \mathrm{~b}$ and 130 were allocated to received hydroxyurea. Three patients in the hydroxyurea group withdrew consent after randomisation and

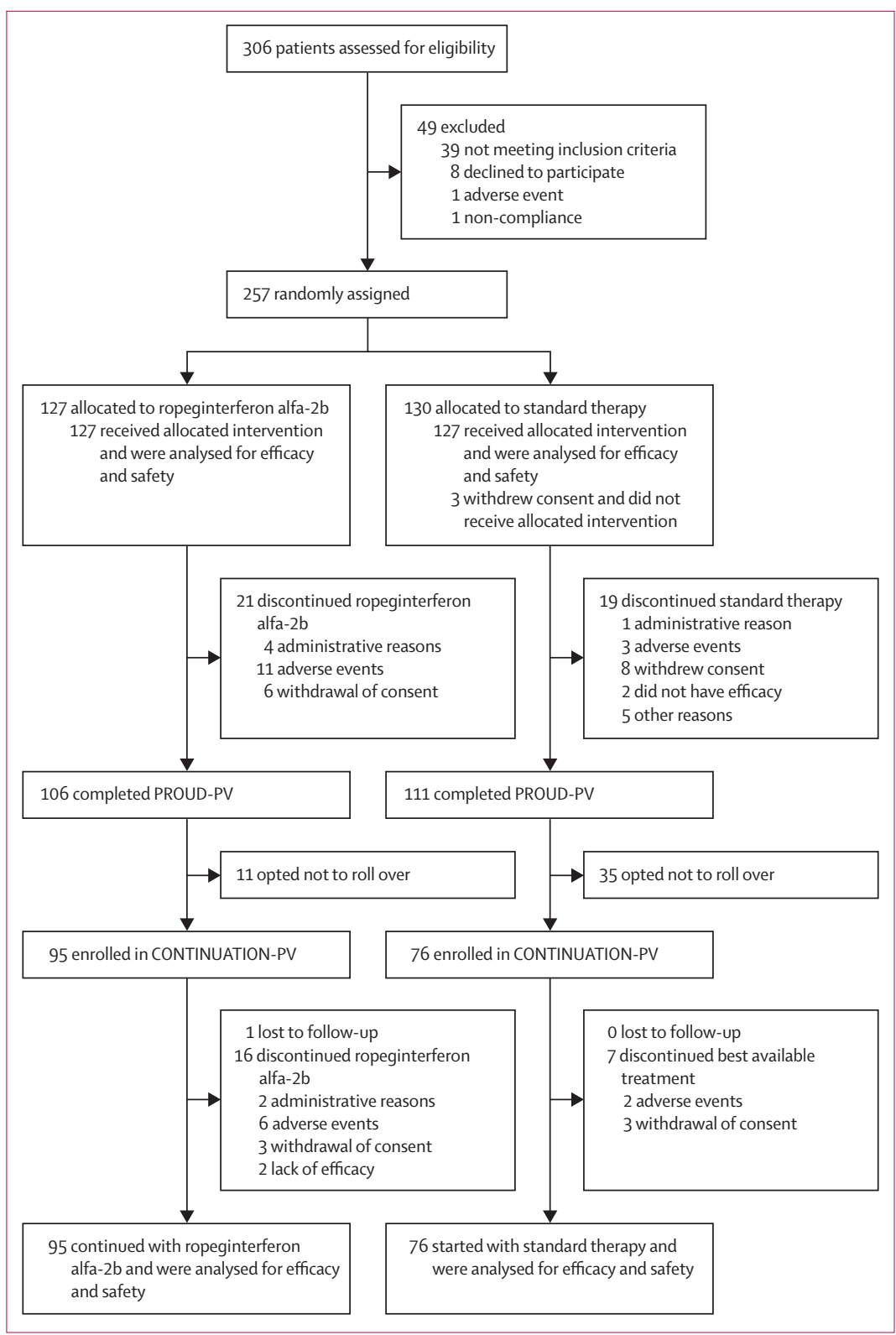

Figure 1: Trial design

finally 127 patients received treatment. A total of 21 (17\%) of 127 in the ropeginterferon alfa-2b group and 16 (13\%) of 127 patients in the hydroxyurea group prematurely discontinued the study after receiving treatment (figure 1). Of the 217 patients who completed the PROUD-PV study, 171 patients rolled over to the CONTINUATION-PV trial. 95 (75\%) of 127 patients continued to receive ropeginterferon alfa- $2 \mathrm{~b}$ and $76(60 \%)$ of 127 patients received best available treatment (hydroxyurea in 64 [97\%] of 66 patients and conventional IFNa in two [3\%] of 66 patients as of month 36). As of database lock in CONTINUATION-PV (May 29, 2018), $17(18 \%)$ of 95 patients in the ropeginterferon group and 


\begin{tabular}{|c|c|c|c|c|}
\hline & \multicolumn{2}{|l|}{ PROUD-PV } & \multicolumn{2}{|l|}{ CONTINUATION-PV* } \\
\hline & Ropeginterferon alfa-2b ( $n=127)$ & Hydroxyurea $(n=127)$ & Ropeginterferon alfa-2b ( $n=95)$ & Best available treatment $(n=76)$ \\
\hline Female & $68(54 \%)$ & $67(53 \%)$ & $48(51 \%)$ & $40(53 \%)$ \\
\hline Male & $59(46 \%)$ & $60(47 \%)$ & $47(49 \%)$ & $36(47 \%)$ \\
\hline \multicolumn{5}{|l|}{ Age, years } \\
\hline Median & $60 \cdot 0(52 \cdot 0-66 \cdot 0)$ & $60 \cdot 0(48 \cdot 0-67 \cdot 0)$ & $58 \cdot 0(50 \cdot 0-64 \cdot 0)$ & $59 \cdot 0(49 \cdot 0-65 \cdot 5)$ \\
\hline Range & $30-85$ & $21-81$ & $30-85$ & $32-79$ \\
\hline Hydroxyurea pretreated & $45(35 \%)$ & $37(29 \%)$ & $30(32 \%)$ & $20(26 \%)$ \\
\hline $\begin{array}{l}\text { Median duration of previous } \\
\text { hydroxyurea therapy, months } \dagger\end{array}$ & $10 \cdot 2(2 \cdot 1-21 \cdot 3)$ & $7 \cdot 9(2 \cdot 7-19 \cdot 2)$ & $9 \cdot 5(2 \cdot 8-25 \cdot 1)$ & $8 \cdot 2(2 \cdot 6-23 \cdot 0)$ \\
\hline $\begin{array}{l}\text { Median duration of } \\
\text { polycythaemia vera, months } \neq\end{array}$ & $1.9(0.7-11 \cdot 2)$ & $3.6(0 \cdot 7-20 \cdot 0)$ & $1 \cdot 8(0 \cdot 6-6 \cdot 8)$ & $1 \cdot 6(0 \cdot 7-15 \cdot 1)$ \\
\hline Previous thromboembolic event & $25(20 \%)$ & $23(18 \%)$ & $21(22 \%)$ & $14(18 \%)$ \\
\hline \multicolumn{5}{|c|}{ Positive status for JAK2 Val617Phe mutation \$ } \\
\hline Number & $126(99 \%)$ & $125(98 \%)$ & $94(99 \%)$ & $74(97 \%)$ \\
\hline Mean allele burden, $\%$ & $41 \cdot 9 \%(24)$ & $42 \cdot 8 \%(24)$ & $42 \cdot 8 \%(23)$ & $42 \cdot 9 \%(23)$ \\
\hline Median haematocrit, \% & $47 \cdot 1 \%(44 \cdot 2-51 \cdot 3)$ & $48 \cdot 0 \%(45 \cdot 0-52 \cdot 2)$ & $47 \cdot 7 \%(44 \cdot 4-52 \cdot 0)$ & $49 \cdot 9 \%(46 \cdot 2-53 \cdot 1)$ \\
\hline Median platelet count, $10^{9} / \mathrm{L}$ & $485 \cdot 0(350 \cdot 0-671 \cdot 0)$ & $452 \cdot 0(329 \cdot 0-666 \cdot 0)$ & $488 \cdot 0(350 \cdot 0-701 \cdot 0)$ & $451 \cdot 0(329 \cdot 0-678 \cdot 5)$ \\
\hline Median leucocyte count, $10^{9} / \mathrm{L}$ & $10 \cdot 6(8 \cdot 0-13 \cdot 4)$ & $10 \cdot 5(7 \cdot 9-14 \cdot 5)$ & $10 \cdot 9(8 \cdot 0-14 \cdot 6)$ & $11 \cdot 3(8 \cdot 7-15 \cdot 1)$ \\
\hline Median spleen size, $\mathrm{cm}$ & $13 \cdot 1(11 \cdot 0-15 \cdot 0)$ & $13 \cdot 0(11 \cdot 5-15 \cdot 2)$ & $13 \cdot 5(11 \cdot 5-15 \cdot 0)$ & $12 \cdot 8(11 \cdot 3-15 \cdot 5)$ \\
\hline Presence of splenomegaly $₫$ & $12(9 \%)$ & $15(12 \%)$ & $7(7 \%)$ & $8(11 \%)$ \\
\hline \multicolumn{5}{|c|}{$\begin{array}{l}\text { Data are } n(\%) \text {, mean (SD), median (IQR). }{ }^{*} \text { Characteristics for the CONTINUATION-PV population at baseline in PROUD-PV. †Duration of previous hydroxyurea therapy was } \\
\text { assessed from start of therapy until the time of screening in PROUD-PV. ¥Duration of polycythaemia vera was assessed from diagnosis until the time of screening in } \\
\text { PROUD-PV. SData were not available for one patient in the ropeginterferon alfa- } 2 \text { b group, and for two patients in the control group in PROUD-PV at baseline. Positive status } \\
\text { for JAK2 Val617Phe mutation was confirmed at subsequent visit. } \uparrow S \text { Splenomegaly as assessed by investigator. }\end{array}$} \\
\hline
\end{tabular}

seven $(9 \%)$ of 76 patients in the standard therapy group had withdrawn.

Baseline characteristics are shown in table 1 for both parts of the study. We did not identify any selection bias regarding enrolment in the extension study: we did not detect any significant differences in patient characteristics or response between patients who rolled over and those who did not (appendix pp 17-20, 23).

Median follow-up in the first year was $52 \cdot 0$ weeks (IQR 51.9-52.1) in the ropeginterferon alfa-2b group and 52.0 weeks $(51 \cdot 9-52 \cdot 1)$ in the hydroxyurea group (PROUD-PV), and median overall follow-up was $182 \cdot 1$ weeks $(166 \cdot 3-201 \cdot 7)$ in the ropeginterferon alfa-2b group and $164 \cdot 5$ weeks $(144 \cdot 4-169 \cdot 3)$ in the hydroxyurea group. Ropeginterferon alfa-2b was uptitrated cautiously in PROUD-PV. The mean efficacious dose was reached after approximately 3.7 months for ropeginterferon alfa-2b $(16 \cdot 2$ weeks [95\% CI 14.8-17·6]) and approximately 2.6 months for hydroxyurea (11.4 weeks [95\% CI 10 -2-12.6]). Initial dosing titration is described in the appendix p 12. At month 36, the median dose per administration of ropeginterferon alfa-2b was $425 \mu \mathrm{g}$ (IQR 250-500) and of and hydroxyurea was $1000 \mathrm{mg}$ (750-1375), remaining stable since month 12 (appendix p 14).

We analysed the 254 patients treated in PROUD-PV for efficacy and safety. For CONTINUATION-PV, we analysed efficacy data up to 36 months for patients enrolled in the extension study $(n=171)$ and all safety data available at database lock ( $\mathrm{n}=254$; includes both studies). For conciseness, secondary endpoints that were analysed as part of a composite outcome were not additionally reported as individual parameters.

In the PROUD-PV study, 26 (21\%) of 122 patients in the ropeginterferon alfa-2b group and 34 (28\%) of 123 patients in the hydroxyurea group met the composite primary endpoint of complete haematological response with normal spleen size at 12 months. The $95 \%$ CI of the difference in response was $-17 \cdot 23$ to $4 \cdot 09$; non-inferiority was not shown $(p=0 \cdot 23)$. For complete haematological response without the spleen criterion at 12 months, responses were similar between the treatment groups (53 [43\%] of 123 patients for ropeginterferon alfa-2b versus 57 [46\%] of 125 patients in the hydroxyurea group; $95 \% \mathrm{CI}$ of difference in responses: -15.55 to $9.52, p=0.63$ ). Hydroxyurea pretreatment had no significant effect on complete haematological response at 12 months. In the ropeginterferon alfa-2b group at 12 months, 18 (39\%) of 46 patients who had been pretreated with hydroxyurea and 35 (46\%) of 77 patients previously untreated with hydroxyurea had a complete haematological response (odds ratio [OR] $0 \cdot 57$ [0.20-1.42], $\mathrm{p}=0 \cdot 24$ ); in the hydroxyurea group, 15 (32\%) of 47 previously treated patients and $42(54 \%)$ of 78 patients previously untreated were responders (OR $0 \cdot 43[0 \cdot 17-1 \cdot 02], \mathrm{p}=0 \cdot 066)$.

In the extension study CONTINUATION-PV, the proportion of patients with a complete haematological response was significantly higher in the ropeginterferon 
alfa-2b group than in the hydroxyurea group (figure 2, table 2). Response kinetics for the composite endpoint complete haematological response and improved disease burden showed a similar pattern to the results for complete haematological response alone: proportion of patients with a response in the ropeginterferon alfa-2b group increased gradually up to 24 months and remained high at 36 months (table 2), whereas in the hydroxyurea group, the response was highest at 12 months and reduced thereafter (table 2). The difference in response between the treatment groups was significant at 36 months (table 2; appendix p 15). A sensitivity analysis in which splenomegaly was defined as spleen length more than $17 \mathrm{~cm}$ supported the results of the original analysis (53 [56\%] of 95 patients for ropeginterferon alfa-2b and 31 [42\%] of 74 patients for hydroxyurea treatment; RR 1.38 [95\% CI 1.01-1.89], $\mathrm{p}=0 \cdot 044)$.

Complete haematological response including normalisation of spleen size among patients treated with ropeginterferon alfa-2b also increased steadily over time, from $27(30 \%)$ of 91 patients at month 12 to 38 (42\%) of 90 patients at month 36. However, no significant difference was reported between the ropeginterferon alfa-2b and hydroxyurea groups (at month 12, 27 [30\%] of 91 and 33 [43\%] of 76, respectively, p=0 .066; at month 24, 34 [37\%] of 91 and 23 [34\%] of 67 , respectively, $p=0.68$; at month 36, 38 [42\%] of 90 and 21 [30\%] of 69, respectively, $\mathrm{p}=0 \cdot 16$; appendix pp 15, 24, 25).

Over the 36-month treatment period, the proportion of patients who maintained their response were significantly higher in the ropeginterferon alfa- $2 b$ group compared with that of the hydroxyurea group regarding complete haematological response as well as complete haematological response with improved disease burden (37 [39\%] of 95 vs 11 [15\%] of 76, p=0.0011; 28 [30\%] of 95 vs $11[15 \%]$ of $76, p=0 \cdot 025$, respectively). The probability of maintaining complete haematological response over time is shown in the appendix $\mathrm{p} 16$.

Molecular responses in PROUD-PV were not significantly different between the treatment groups at 12 months (42 [34\%] of 123 in the ropeginterferon alfa-2b groups vs 52 [42\%] of 123 in the hydroxyurea group; $\mathrm{p}=0 \cdot 19$; appendix $\mathrm{p} 27$ ). In both groups, a reduction in mean JAK2 Val617Phe allele burden compared with baseline was evident at 12 months (from 41.9\%

Figure 2: Complete haematological response and molecular response in the PROUD-PV and CONTINUATION-PV studies Error bars show standard deviation of the response at each timepoint assessed. A) Proportion of patients with complete haematological response over 12 months of treatment during the PROUD-PV study. B) Proportion of patients with complete haematological response during a treatment period of 36 months among patients who participated in the extension study, CONTINUATION-PV. C) The proportion of patients with molecular response during a treatment period of 36 months in CONTINUATION-PV. ${ }^{*} \mathrm{p}<0.05$ $+\mathrm{p}<0.01$
[SD $23 \cdot 49]$ to $30 \cdot 7 \%$ [SD $22 \cdot 66$ ] in the ropeginterferon alfa-2b group and from $42.8 \%$ [SD 24.14] to $25.9 \%$ [SD 21.49] in the hydroxyurea group; appendix p 26). At 24 months and 36 months of treatment, assessed in the
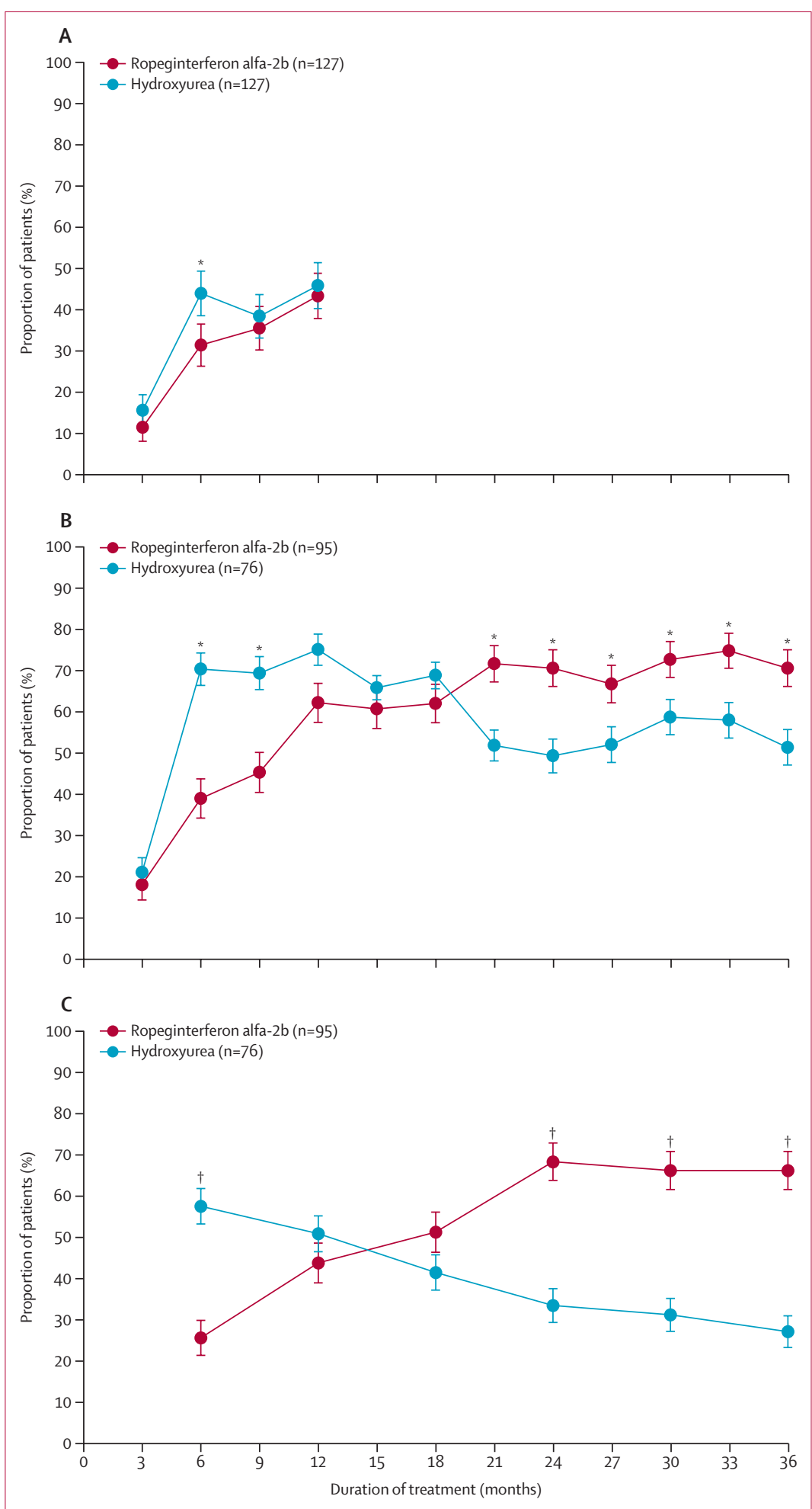


\begin{tabular}{|c|c|c|c|c|c|}
\hline & $\begin{array}{l}\text { Ropeginterferon } \\
\text { alfa-2b }\end{array}$ & Control & $\begin{array}{l}\text { Difference in responses } \\
(95 \% \mathrm{Cl})\end{array}$ & Rate ratio $(95 \% \mathrm{Cl})$ & p value \\
\hline \multicolumn{6}{|l|}{ PROUD-PV ( $n=254)$} \\
\hline $\begin{array}{l}\text { Complete haematological response* } \\
\text { and normal spleen size† at month } 12\end{array}$ & $26 / 122(21 \%)$ & $34 / 123(28 \%)$ & $-6 \cdot 57(-17 \cdot 23$ to $4 \cdot 09)$ & .. & 0.23 \\
\hline $\begin{array}{l}\text { Complete haematological response } \\
\text { at month } 12\end{array}$ & $53 / 123(43 \%)$ & $57 / 125(46 \%)$ & $-3.02(-15.55$ to 9.52$)$ &.. & 0.63 \\
\hline Molecular response $\ddagger$ at month 12 & $42 / 123(34 \%)$ & $52 / 123(42 \%)$ & $-8.07(-19.99$ to 3.84$)$ & .. & 0.19 \\
\hline \multicolumn{6}{|l|}{ CONTINUATION-PV (n=171) } \\
\hline \multicolumn{6}{|c|}{ Complete haematological response and improvement in disease burden $\S$} \\
\hline Month 12 & $44 / 95(46 \%)$ & $39 / 76(51 \%)$ &.$\cdot$ & $0.91(0.67$ to 1.23$)$ & 0.52 \\
\hline Month 24 & $47 / 95(50 \%)$ & $27 / 71(38 \%)$ & .. & $1.27(0.89$ to 1.81$)$ & 0.18 \\
\hline Month 36 & $50 / 95(53 \%)$ & 28/74 (38\%) &.. & $1.42(1.01$ to 2.00$)$ & 0.044 \\
\hline \multicolumn{6}{|l|}{ Complete haematological response } \\
\hline Month 12 & $59 / 95(62 \%)$ & $57 / 76(75 \%)$ & .. & $0.85(0.70$ to 1.04$)$ & 0.12 \\
\hline Month 24 & $67 / 95(71 \%)$ & $33 / 67(49 \%)$ &.$\cdot$ & $1.42(1.08$ to 1.86$)$ & 0.011 \\
\hline Month 36 & $67 / 95(71 \%)$ & $38 / 74(51 \%)$ &.. & 1.38 (1.07 to 1.79$)$ & 0.012 \\
\hline \multicolumn{6}{|l|}{ Molecular response } \\
\hline Month 12 & $41 / 94(44 \%)$ & $38 / 75(51 \%)$ & .. & $0.84(0.62$ to 1.15$)$ & 0.29 \\
\hline Month 24 & $64 / 94(68 \%)$ & $25 / 75(33 \%)$ & .. & $1.94(1.38$ to 2.72$)$ & 0.0001 \\
\hline Month 36 & $62 / 94(66 \%)$ & $20 / 74(27 \%)$ &.. & $2 \cdot 31$ (1.56 to 3.42$)$ & $<0.0001$ \\
\hline \multicolumn{6}{|c|}{$\begin{array}{l}\text { Data are } \mathrm{n} / \mathrm{N}(\%) \text {. *Complete haematological response was defined as haematocrit }<45 \% \text { without phlebotomy (at least } 3 \text { months since the last phlebotomy), platelet count } \\
<400 \times 10^{9} / \mathrm{L} \text { and leucocyte count }<10 \times 10^{9} / \mathrm{L} \text {. Haematological parameters were measured centrally in PROUD-PV. Local laboratory values were used throughout } \\
\text { CONTINUATION-PV. } \dagger \text { Normal spleen size was defined as a longitudinal diameter of } \leq 12 \mathrm{~cm} \text { for women and } \leq 13 \mathrm{~cm} \text { for men. } \neq \text { Molecular response was calculated from JAK2 } \\
\text { Val617Phe allele burden values. Molecular response was defined as reduction of any molecular abnormality to undetectable levels (complete molecular response) or a } \\
\text { reduction from baseline value of } \geq 50 \% \text { in patients with }<50 \% \text { JAK2 Val } 617 \text { Phe allele burden at baseline; a reduction from baseline value of } \geq 25 \% \text { in patients with } \geq 50 \% \text { JAK2 } \\
\text { Val } 617 \text { Phe allele burden at baseline (partial molecular response). A partial molecular response applied only to patients with a baseline value of JAK } 2 \text { Val } 617 \text { Phe allele } \\
\text { burden }>10 \% \text {. Missing outcomes from patients were imputed using last observation carried forward method. SDisease burden was defined as disease-related signs } \\
\text { (clinically significant splenomegaly) and symptoms (microvascular disturbances and headache) as assessed by the investigator. }\end{array}$} \\
\hline
\end{tabular}

\begin{tabular}{|c|c|c|}
\hline & Ropeginterferon alfa-2b $(\mathrm{N}=127)$ & Control $(\mathrm{N}=127)$ \\
\hline \multicolumn{3}{|l|}{ Endocrine disorders } \\
\hline Any adverse event & $8(6 \%)$ & $2(2 \%)$ \\
\hline Related to treatment & $6(5 \%)$ & 0 \\
\hline \multicolumn{3}{|l|}{ Psychiatric disorders } \\
\hline Any adverse event & $5(4 \%)$ & $6(5 \%)$ \\
\hline Related to treatment & $2(2 \%)$ & $1(1 \%)$ \\
\hline \multicolumn{3}{|l|}{ Musculoskeletal and connective tissue disorders } \\
\hline Any adverse event & $2(2 \%)$ & 0 \\
\hline Related to treatment & $2(2 \%)$ & 0 \\
\hline \multicolumn{3}{|c|}{ Major cardiovascular and major thromboembolic adverse events } \\
\hline Any major cardiovascular adverse event & $13(10 \%) ; 16$ events & $8(6 \%) ; 25$ events \\
\hline Major thromboembolic adverse event & $4(3 \%) ; 6$ events & $4(3 \%) ; 4$ events \\
\hline \multicolumn{3}{|c|}{ Neoplasms benign, malignant and unspecified (including cysts and polyps) } \\
\hline Any neoplasm & $9(7 \%) ; 11$ events & $10(8 \%) ; 12$ events \\
\hline Leukaemic transformation (acute leukaemia) & $0 ; 0$ events & $2(2 \%) ; 2$ events \\
\hline $\begin{array}{l}\text { Skin cancers related to treatment (basal cell } \\
\text { carcinoma and melanoma) }\end{array}$ & $0 ; 0$ events & $3(2 \%) ; 3$ events \\
\hline
\end{tabular}

CONTINUATION-PV study, the numbers of patients with a molecular response were significantly higher in the ropeginterferon alfa- $2 b$ group than in the hydroxyurea group (figure 2, table 2). Patients treated

with ropeginterferon alfa- $2 \mathrm{~b}$ showed a steady decrease in the mean absolute JAK2 Val617Phe allele burden to less than half the baseline level by month 36 (from $42 \cdot 8 \%$ [SD 23.4] to $19 \cdot 7 \%$ [SD 21.3]) whereas in the hydroxyurea group the reduction was transient and was lost by month $36(42.9 \%$ [SD 23.0] at baseline and $39.3 \%$ [SD 25.91] at month 36; appendix $p$ 28). At months 24 and 36, mean JAK2 Val617Phe allele burden was significantly lower among patients treated with ropeginterferon alfa- $2 \mathrm{~b}$ compared with the hydroxyurea group (at month 24, 20 -9\% [SD 20.8] and 32.1\% [23.4], respectively, $\mathrm{p}<0 \cdot 0001$; at month $36,19 \cdot 7 \%$ [21.3] and $39.3 \%$ [25.9], respectively; $\mathrm{p}<0 \cdot 0001)$.

In a post-hoc analysis, lower JAK2 Val617Phe allele burden correlated with complete haematological response at 12 months, 24 months, and 36 months in the ropeginterferon alfa- $2 \mathrm{~b}$ group (Pearson correlation -0.45 $[\mathrm{p}<0.0001],-0.24 \quad[\mathrm{p}=0.037]$, and $-0.31 \quad[\mathrm{p}=0.009]$, respectively; appendix p 29).

Bone marrow histology at baseline, 12 months, and end of treatment was prospectively planned; however, only a seven patients consented to this optional assessment and no meaningful conclusions could be drawn from the available results.

The cohort studied represents an early polycythaemia vera population with a median duration since diagnosis 
of only a few months $(1.9$ months in the ropeginterferon alfa- $2 \mathrm{~b}$ group, 3.6 months in the standard therapy group), although patients with both no history of cytoreduction and those pretreated with hydroxyurea were included (45 [35\%] of 127 hydroxyurea pretreated patients in the ropeginterferon alfa-2b group, 37 [29\%] of 127 hydroxyurea pretreated in the standard therapy group). Consequently, almost half of the patients (59 [47\%] of 127 in both study groups) had normal spleen size at baseline; most of the remaining patients had modestly enlarged spleens and only very few patients presented with splenomegaly (12 [9\%] of 127 patients in the ropeginterferon alfa- $2 \mathrm{~b}$ group; 15 [12\%] of 127 in the standard therapy group).

Quality of life data collected using the EQ-5D-3L questionnaire showed no differences between the groups at month 36 . For the ropeginterferon alfa- $2 \mathrm{~b}$ and standard therapy treatment groups respectively, the mean changes from baseline were $-0 \cdot 0$ (SD 1.1) and $-0 \cdot 1$ (SD 1.5) for the total score and 1.6 (SD 13.0) and 1.2 (SD 14.4) for the visual analogue score.

Adverse events required dose reduction in 51 (40\%) of 127 patients in the ropeginterferon alfa- $2 \mathrm{~b}$ group and $74(58 \%)$ of 127 patients in the standard therapy group, and dose interruption in 29 (23\%) of 127 patients and $23(18 \%)$ of 127 patients, respectively. 11 patients $(8 \%)$ of 127 in the ropeginterferon alfa- $2 \mathrm{~b}$ group discontinued due to drug-related toxicity (thrombocytopenia in one patient, hypothyroidism in two patients, dyspnoea and pneumonitis in one patient, increased aspartate aminotransferase and alanine aminotransferase in one patient, microcytic anaemia and anaemia in one patient, and anxiety, psoriasis, depression, Sjögren's syndrome, and rheumatoid arthritis in one patient each). In the standard therapy group across both studies, five (4\%) of 127 patients discontinued as a result of drug-related toxicity: thrombocytopenia and anaemia in one patient, and basal cell carcinoma, pyrexia, skin ulcer, and intolerance to hydroxyurea in one patient each.

The most commonly reported adverse events (in $>10 \%$ of patients) in the 127 patients in the ropeginterferon alfa- $2 \mathrm{~b}$ group were thrombocytopenia (28 [22\%]), leucopenia (25 [20\%]), increased $\gamma$-glutamyltransferase (24 [19\%]), fatigue (17 [13\%]), increased alanine aminotransferase (17 [13\%]), anaemia (16 [13\%]), increased aspartate aminotransferase (13 [10\%]), headache (15 [12\%]), arthralgia (15 [12\%]), and dizziness (14 [11\%]).

The most commonly reported adverse events in the 127 patients in the standard therapy group were thrombocytopenia (37 [29\%]), anaemia (32 [25\%]), leucopenia (29 [23\%]), fatigue (18 [14\%]), headache (16 [13\%]), nausea (15 [12\%]), diarrhoea (14 [11\%]), and nasopharyngitis (13 [10\%]).

Adverse events considered by the investigator to be treatment related were reported in 95 (75\%) of 127 patients in the ropeginterferon alfa-2b group and in 100 (79\%) of 127 patients in the standard therapy group (appendix p 30).
The proportion of serious adverse events and adverse events with a severity of grade three or higher were similar in both treatment groups. Treatment-related serious adverse events occurred in three (2\%) of 127 patients in the ropeginterferon alfa-2b group (atrial fibrillation, microcytic anaemia and anaemia, and rheumatoid arthritis) and

\begin{tabular}{|c|c|c|c|c|c|c|}
\hline & \multicolumn{3}{|c|}{ Ropeginterferon alfa-2b ( $n=127)$} & \multicolumn{3}{|c|}{ Control $(n=127)$} \\
\hline & Grade 1-2* & Grade 3 & Grade 4 & Grade 1-2 & Grade 3 & Grade 4 \\
\hline Any adverse event & $113(89 \%)$ & $40(32 \%)$ & $3(2 \%)$ & $114(90 \%)$ & $33(26 \%)$ & $1(1 \%)$ \\
\hline Thrombocytopenia & $27(21 \%)$ & $3(2 \%)$ & 0 & $36(28 \%)$ & $5(4 \%)$ & 0 \\
\hline Leucopenia & $23(18 \%)$ & $3(2 \%)$ & 0 & $28(22 \%)$ & $6(5 \%)$ & 0 \\
\hline Anaemia & $16(13 \%)$ & $1(1 \%)$ & 0 & $31(24 \%)$ & $2(2 \%)$ & 0 \\
\hline Fatigue & $17(13 \%)$ & 0 & 0 & $17(13 \%)$ & $1(1 \%)$ & 0 \\
\hline $\begin{array}{l}\gamma \text {-glutamyltransferase } \\
\text { increased }\end{array}$ & $20(16 \%)$ & $9(7 \%)$ & $1(1 \%)$ & $2(2 \%)$ & $2(2 \%)$ & 0 \\
\hline Headache & $15(12 \%)$ & 0 & 0 & $16(13 \%)$ & 0 & 0 \\
\hline Diarrhoea & $12(9 \%)$ & 0 & 0 & $14(11 \%)$ & $1(1 \%)$ & 0 \\
\hline Dizziness & $14(11 \%)$ & 0 & 0 & $10(8 \%)$ & 0 & 0 \\
\hline $\begin{array}{l}\text { Alanine aminotransferase } \\
\text { increased }\end{array}$ & $16(13 \%)$ & $5(4 \%)$ & 0 & $2(2 \%)$ & 0 & 0 \\
\hline Arthralgia & $15(12 \%)$ & $1(1 \%)$ & 0 & $5(4 \%)$ & 0 & 0 \\
\hline Hypertension & $5(4 \%)$ & $4(3 \%)$ & 0 & $6(5 \%)$ & $5(4 \%)$ & 0 \\
\hline Nasopharyngitis & $7(6 \%)$ & 0 & 0 & $13(10 \%)$ & 0 & 0 \\
\hline Nausea & $4(3 \%)$ & 0 & 0 & $15(12 \%)$ & 0 & 0 \\
\hline $\begin{array}{l}\text { Aspartate aminotransferase } \\
\text { increased }\end{array}$ & $13(10 \%)$ & $3(2 \%)$ & 0 & $2(2 \%)$ & 0 & 0 \\
\hline Asthenia & $10(8 \%)$ & 0 & 0 & $6(5 \%)$ & $1(1 \%)$ & 0 \\
\hline Platelet count decreased & $3(2 \%)$ & 0 & 0 & $12(9 \%)$ & $2(2 \%)$ & 0 \\
\hline Pain in extremity & $10(8 \%)$ & $1(1 \%)$ & 0 & $5(4 \%)$ & 0 & 0 \\
\hline Abdominal pain & $8(6 \%)$ & 0 & 0 & $7(6 \%)$ & $1(1 \%)$ & 0 \\
\hline Influenza & $4(3 \%)$ & $2(2 \%)$ & 0 & $10(8 \%)$ & $2(2 \%)$ & 0 \\
\hline Urinary tract infection & $8(6 \%)$ & $1(1 \%)$ & 0 & $5(4 \%)$ & 0 & 0 \\
\hline Neutropenia & $3(2 \%)$ & $2(2 \%)$ & 0 & $4(3 \%)$ & $2(2 \%)$ & 0 \\
\hline Cataract & $7(6 \%)$ & 0 & 0 & $3(2 \%)$ & $1(1 \%)$ & 0 \\
\hline $\begin{array}{l}\text { White blood cell count } \\
\text { decreased }\end{array}$ & $5(4 \%)$ & 0 & 0 & $4(3 \%)$ & $1(1 \%)$ & 0 \\
\hline Hepatic enzyme increased & $7(6 \%)$ & $1(1 \%)$ & 0 & $1(1 \%)$ & 0 & 0 \\
\hline Anxiety & $4(3 \%)$ & $1(1 \%)$ & 0 & $3(2 \%)$ & 0 & 0 \\
\hline Atrial fibrillation & $5(4 \%)$ & 0 & 0 & $2(2 \%)$ & $1(1 \%)$ & 0 \\
\hline Cholelithiasis & $3(2 \%)$ & $1(1 \%)$ & 0 & $3(2 \%)$ & 0 & 0 \\
\hline Hypocalcaemia & $3(2 \%)$ & $1(1 \%)$ & 0 & $2(2 \%)$ & 0 & 0 \\
\hline Osteoarthritis & $4(3 \%)$ & 0 & 0 & $1(1 \%)$ & $1(1 \%)$ & 0 \\
\hline Depression & $3(2 \%)$ & 0 & 0 & $2(2 \%)$ & $1(1 \%)$ & 0 \\
\hline $\begin{array}{l}\text { Blood lactate dehydrogenase } \\
\text { increased }\end{array}$ & $3(2 \%)$ & $1(1 \%)$ & 0 & $1(1 \%)$ & 0 & 0 \\
\hline Platelet count increased & $2(2 \%)$ & $1(1 \%)$ & 0 & $2(2 \%)$ & 0 & 0 \\
\hline Cataract operation & 0 & 0 & 0 & $4(3 \%)$ & $1(1 \%)$ & 0 \\
\hline Stomatitis & $2(2 \%)$ & 0 & 0 & $2(2 \%)$ & $1(1 \%)$ & 0 \\
\hline Hypokalaemia & $2(2 \%)$ & $1(1 \%)$ & 0 & $1(1 \%)$ & 0 & 0 \\
\hline Neck pain & $2(2 \%)$ & 0 & 0 & 0 & $1(1 \%)$ & 0 \\
\hline Granulocytopenia & $1(1 \%)$ & 0 & 0 & $1(1 \%)$ & $1(1 \%)$ & 0 \\
\hline Psoriasis & $1(1 \%)$ & $1(1 \%)$ & 0 & $1(1 \%)$ & 0 & 0 \\
\hline \multirow[t]{2}{*}{ Cardiac failure } & 0 & 0 & 0 & $1(1 \%)$ & $1(1 \%)$ & 0 \\
\hline & & & & \multicolumn{3}{|c|}{ (Table 4 continues on next page) } \\
\hline
\end{tabular}




\begin{tabular}{|c|c|c|c|c|c|c|}
\hline & \multicolumn{3}{|c|}{ Ropeginterferon alfa- $2 b(n=127)$} & \multicolumn{3}{|c|}{ Control $(n=127)$} \\
\hline & Grade $1-2^{*}$ & Grade 3 & Grade 4 & Grade 1-2 & Grade 3 & Grade 4 \\
\hline \multicolumn{7}{|l|}{ (Continued from previous page) } \\
\hline Blood bilirubin increased & 0 & 0 & 0 & $1(1 \%)$ & $1(1 \%)$ & 0 \\
\hline Microcytic anaemia & $1(1 \%)$ & $1(1 \%)$ & 0 & 0 & 0 & 0 \\
\hline Fall & $1(1 \%)$ & $1(1 \%)$ & 0 & 0 & 0 & 0 \\
\hline Basal cell carcinoma & 0 & 0 & 0 & $1(1 \%)$ & $1(1 \%)$ & 0 \\
\hline Blood pressure increased & 0 & $1(1 \%)$ & 0 & $1(1 \%)$ & 0 & 0 \\
\hline Sinus tachycardia & $1(1 \%)$ & $1(1 \%)$ & 0 & 0 & 0 & 0 \\
\hline Arthritis reactive & $1(1 \%)$ & $1(1 \%)$ & 0 & 0 & 0 & 0 \\
\hline Adrenal adenoma & 0 & $1(1 \%)$ & 0 & $1(1 \%)$ & 0 & 0 \\
\hline Lipase increased & $1(1 \%)$ & $1(1 \%)$ & 0 & 0 & 0 & 0 \\
\hline Ischaemic stroke & $1(1 \%)$ & $1(1 \%)$ & 0 & 0 & 0 & 0 \\
\hline $\begin{array}{l}\text { Peripheral arterial occlusive } \\
\text { disease }\end{array}$ & $1(1 \%)$ & $1(1 \%)$ & 0 & 0 & 0 & 0 \\
\hline Multiorgan failure & 0 & 0 & $1(1 \%)$ & 0 & 0 & 0 \\
\hline Cholecystitis acute & 0 & 0 & $1(1 \%)$ & 0 & 0 & 0 \\
\hline Cholangitis infective & 0 & 0 & $1(1 \%)$ & 0 & 0 & 0 \\
\hline Hyponatraemia & 0 & 0 & 0 & 0 & 0 & $1(1 \%)$ \\
\hline Septic shock & 0 & 0 & $1(1 \%)$ & 0 & 0 & 0 \\
\hline Bile duct cancer & 0 & 0 & $1(1 \%)$ & 0 & 0 & 0 \\
\hline Acute kidney injury & 0 & 0 & $1(1 \%)$ & 0 & 0 & 0 \\
\hline Acute leukaemia & 0 & 0 & 0 & 0 & $1(1 \%)$ & 0 \\
\hline Appendicitis & 0 & $1(1 \%)$ & 0 & 0 & 0 & 0 \\
\hline Skull fractured base & 0 & $1(1 \%)$ & 0 & 0 & 0 & 0 \\
\hline Pyelonephritis acute & 0 & 0 & 0 & 0 & $1(1 \%)$ & 0 \\
\hline Neoplasm of appendix & 0 & 0 & 0 & 0 & $1(1 \%)$ & 0 \\
\hline Diabetic neuropathy & 0 & 0 & 0 & 0 & $1(1 \%)$ & 0 \\
\hline Upper limb fracture & 0 & 0 & 0 & 0 & $1(1 \%)$ & 0 \\
\hline Femoral artery occlusion & 0 & 0 & 0 & 0 & $1(1 \%)$ & 0 \\
\hline $\begin{array}{l}\text { Diabetic metabolic } \\
\text { decompensation }\end{array}$ & 0 & $1(1 \%)$ & 0 & 0 & 0 & 0 \\
\hline $\begin{array}{l}\text { Eastern Cooperative Oncology } \\
\text { Group performance status } \\
\text { worsened }\end{array}$ & 0 & $1(1 \%)$ & 0 & 0 & 0 & 0 \\
\hline Cardiac failure acute & 0 & 0 & 0 & 0 & $1(1 \%)$ & 0 \\
\hline Uterine dehiscence & 0 & 0 & 0 & 0 & $1(1 \%)$ & 0 \\
\hline Carotid endarterectomy & 0 & 0 & 0 & 0 & $1(1 \%)$ & 0 \\
\hline Umbilical haematoma & 0 & $1(1 \%)$ & 0 & 0 & 0 & 0 \\
\hline Pericardial effusion & 0 & 0 & 0 & 0 & $1(1 \%)$ & 0 \\
\hline Shoulder arthroplasty & 0 & $1(1 \%)$ & 0 & 0 & 0 & 0 \\
\hline Encephalopathy & 0 & $1(1 \%)$ & 0 & 0 & 0 & 0 \\
\hline Hypertensive crisis & 0 & 0 & 0 & 0 & $1(1 \%)$ & 0 \\
\hline Aortic valve replacement & 0 & 0 & 0 & 0 & $1(1 \%)$ & 0 \\
\hline $\begin{array}{l}\text { Bladder transitional cell } \\
\text { carcinoma }\end{array}$ & 0 & $1(1 \%)$ & 0 & 0 & 0 & 0 \\
\hline Colectomy & 0 & 0 & 0 & 0 & $1(1 \%)$ & 0 \\
\hline Knee arthroplasty & 0 & 0 & 0 & 0 & $1(1 \%)$ & 0 \\
\hline Calculus urinary & 0 & 0 & 0 & 0 & $1(1 \%)$ & 0 \\
\hline \multicolumn{7}{|c|}{$\begin{array}{l}\text { Number of patients are reported in whom a given adverse event occurred at least once. *The severity of adverse events } \\
\text { was graded in accordance with the National Cancer Institute Common Terminology Criteria for Adverse Events, } \\
\text { version 4.0. Grade 1-2 events occurring in }>10 \% \text { of patients and all grade } 3 \text { and } 4 \text { events are reported. Two patients in } \\
\text { each treatment group died during the studies: in the ropeginterferon alfa-2b group, one death was attributed to } \\
\text { glioblastoma and one to an unspecified illness other than polycythaemia vera; in the control treatment group, } \\
\text { one death was attributed to treatment-related acute leukaemia and concurrent polycythaemia vera-related } \\
\text { pneumonia and sepsis, and the other death was attributed to polycythaemia vera. }\end{array}$} \\
\hline
\end{tabular}

five (4\%) of 127 patients in the standard therapy group (basal cell carcinoma in two patients; anaemia, leucopenia, and granulocytopenia in one patient; malignant melanoma in one patient; and acute leukaemia in one patient). The case of acute leukaemia considered as hydroxyurea related (together with concurrent pneumonia and sepsis unrelated to treatment) was fatal. No other treatment-related deaths occurred. Three further deaths were recorded, all unrelated to treatment: in the ropeginterferon alfa- $2 \mathrm{~b}$ group, one death was attributed to glioblastoma and one to an unspecified illness other than polycythaemia vera; in the standard therapy group, one death was related to polycythaemia vera.

Major polycythaemia vera-related cardiovascular adverse events were reported both in the ropeginterferon alfa-2b group (16 events in 13 [10\%] of 127 patients) and in the standard therapy group (25 events in eight [6\%] of 127 patients). Two events were considered related to ropeginterferon alfa- $2 \mathrm{~b}$ by the investigator: atrial fibrillation and intracardiac thrombus (in patients who had a history of hypertension but not of thromboembolic events). The frequency of major thromboembolic adverse events were similar between the ropeginterferon alfa- $2 \mathrm{~b}$ group and the standard therapy group (table 3 ). The most frequently reported grade 3 and grade 4 treatment-related adverse events were increased $\gamma$-glutamyltransferase (seven [6\%] of 127 patients) and increased alanine aminotransferase (four [3\%] of 127 patients) in the ropeginterferon alfa-2b group, and leucopenia (six [5\%] of 127 patients) and thrombocytopenia (five [4\%] of 127 patients) in the standard therapy group. Treatmentrelated serious adverse events occurred in three $(2 \%)$ of 127 patients in the ropeginterferon alfa-2b group and five (4\%) of 127 patients in the hydroxyurea group. One treatment-related death was reported in the standard therapy group (acute leukaemia; table 4).

Although the frequency of reported neoplasms were similar in the ropeginterferon alfa-2b (nine [7\%] of 127) and the standard therapy group (ten [8\%] of 127), leukaemia and treatment-related skin cancers occurred only in the standard therapy group (in two [2\%] of 127 patients and three [2\%] of 127 patients, respectively; table 3, table 4). Two cases of acute myeloid leukaemia developed in patients receiving continuous doses of $1000 \mathrm{mg}$ and $750 \mathrm{mg}$ hydroxyurea, respectively; both patients were previously untreated with hydroxyurea before being randomly assigned and the time of onset was 2.3 years and $7 \cdot 1$ years after diagnosis of polycythaemia vera and 1.9 years and 1.6 years after the first administration of hydroxyurea. Further, treatment-related basal cell carcinoma (two patients) and melanoma (one patient) were reported.

\section{Discussion}

To our knowledge, the randomised, controlled PROUD-PV trial and its extension, CONTINUATION-PV, provide the first and largest comparison of an interferon versus 
hydroxyurea or best available treatment in polycythaemia vera, and were the basis of the European Commission's approval of ropeginterferon alfa- $2 \mathrm{~b}$ as a first-line treatment of polycythaemia vera. A smaller randomised, controlled, phase 3 trial comparing IFN $\alpha$ with hydroxyurea has been done in patients with polycythaemia vera or essential thrombocytopenia; however, only patients at high risk were included, and the full results have not yet been reported. ${ }^{28}$

Data from 3 years of treatment confirmed that therapy with ropeginterferon alfa- $2 \mathrm{~b}$ is feasible, safe, and welltolerated in patients with polycythaemia vera. Higher haematological, clinical, and molecular responses and longer response duration compared with the standard therapy were achieved during ropeginterferon alfa- $2 \mathrm{~b}$ treatment assessed at 36 months in CONTINUATION-PV. Response kinetics differed markedly between the two treatment groups: the hydroxyurea or best available treatment group reached maximal responses after only 6 months of treatment, gradually declining thereafter, whereas ropeginterferon alfa- $2 \mathrm{~b}$ responses increased steadily with time. After 1 year of treatment in the PROUD-PV study, the haematological response to ropeginterferon alfa- $2 \mathrm{~b}$ was similar to standard therapy but the composite primary endpoint including both complete haematological response and normalisation of spleen size did not meet the criteria for non-inferiority. By contrast, after 3 years of treatment, the proportion of patients who achieved a haematological response alone and a haematological response with improved disease burden was higher in the ropeginterferon alfa-2b group.

A limitation of these studies was the low number of patients who presented with splenomegaly at baseline, which precluded a clinically meaningful assessment of the spleen response to treatment. A further limitation of the present studies is the scarcity of pathological response data in support of haematological and clinical efficacy outcomes, because very few patients consented to the optional bone marrow assessment. Limitations are also posed by the design of the extension study: statistical power was not recalculated after the initial 12 months, and although the results indicate that the treatments groups were balanced after roll-over, selection bias cannot entirely be ruled out.

Our findings corroborate the disease-modifying potential of IFN $\alpha$-based therapy that has consistently been shown in myeloproliferative neoplasms in several independent phase 2 studies. ${ }^{9,12,20,29}$ The pleiotropic activity of IFN $\alpha$ includes antiproliferative, immunomodulatory, anti-inflammatory, and antiangiogenic properties. ${ }^{4}$ The mechanism of action in polycythaemia vera is probably multimodal: selective exhaustion of haematopoietic stem cells carrying the oncogenic JAK2 mutation is proposed to be driven by differentiation, and the resulting sustained decrease in mutant JAK2 allele burden decreases genetic instability and the propensity to acquire secondary mutations which ultimately contribute to disease progression. ${ }^{30-32}$ In the present trial, the mutant JAK2 allele burden began to rebound during year 2 of hydroxyurea treatment and had returned to baseline levels by month 36 . By contrast, with ropeginterferon alfa- $2 \mathrm{~b}$, molecular responses increased further and the allele burden decreased during years 2 and 3 of treatment to half of baseline levels; additionally, in agreement previous findings in patients treated with ropeginterferon alfa- $2 \mathrm{~b},{ }^{15}$ lower allele burden correlated with haematologic response in the present study. Furthermore, in ancillary studies in patients from the PROUD-PV and CONTINUATION-PV cohort, ex-vivo bone marrow colony assays showed an increased proportion of wild type to mutant colonies after ropeginterferon alfa- $2 \mathrm{~b}$ treatment ${ }^{16}$ and a comprehensively applied next generation sequencing and single nucleotide polymorphism analysis suggested that ropeginterferon alfa-2b, but not hydroxyurea, reduced the non-JAK2 allele burden. ${ }^{33}$ Taken together, these data provide further evidence of a possible disease-modifying activity of IFN $\alpha$ based treatment in myeloproliferative neoplasms.

The use of non-pegylated IFN $\alpha$ in myeloproliferative neoplasms has previously been limited by their toxicity. Our trial shows that ropeginterferon alfa- $2 \mathrm{~b}$, a novel, monopegylated IFN $\alpha$, was well tolerated in patients with polycythaemia vera, and the frequency of treatmentrelated adverse events was similar to that of hydroxyurea. As expected for this cohort of patients with early polycythaemia vera managed optimally in the setting of a prospective trial, only very few major cardiovascular polycythaemia vera-related events were recorded. A relationship between molecular response, reflecting the exhaustion of malignant cells, and long-term benefits in terms of reduction of vascular events in patients with polycythaemia vera has been proposed..$^{34}$ The higher proportion of response among patients treated with ropeginterferon alfa- $2 \mathrm{~b}$ in the second and third year might therefore be expected to be accompanied by a lower rate of thromboembolic events. This effect could not be confirmed by a statistical comparison in the present studies due to the low number of events in both groups. Longer follow-up in this cohort will be important to explore this possible correlation.

Two cases of acute myeloid leukaemia, two basal cell carcinomas, and one melanoma were recorded, all in the hydroxyurea cohort. One case of acute myeloid leukaemia was reported by the investigator as being treatment related. Although the leukaemogenic potential of hydroxyurea has been reported, the topic remains controversial, ${ }^{35}$ and the cause of this individual case cannot be verified. Autoimmune and psychiatric events were followed up with scrutiny. One case of autoimmune thyroiditis and only a few cases of treatment-related psychiatric disorders (in two [2\%] of 127 patients; anxiety in one patient and altered mood, depression and anxiety in one patient) were recorded in the ropeginterferon alfa-2b group, highlighting the excellent tolerability of an interferon treatment. 
In conclusion, ropeginterferon alfa- $2 \mathrm{~b}$ offers a new treatment option for patients with polycythaemia vera that has greater benefits than standard therapy with hydroxyurea after the second year of exposure, suggesting that ropeginterferon alfa- $2 \mathrm{~b}$ treatment should be considered as early as possible in the course of disease.

\section{Contributors}

HG contributed to the study design, collection of clinical data, analysis, and interpretation of data, and the drafting and editing of the manuscript. CK, KK, BG-I, and J-JK contributed to the study design, analysis, interpretation of data, and preparation of the manuscript. PG, DK, LG-K, ME, VR, PD, AI, HP, LS, JM, VY, J-JK, and the PROUD-PV Study Group collected clinical data. RK provided critical analytical tools, oversaw analyses, and interpreted the data. $\mathrm{HCH}$ contributed to interpretation of the data and preparation of the manuscript. We received editorial support from a medical writer funded by AOP Orphan Pharmaceuticals AG.

\section{Declaration of interests}

HG reports grants and personal fees from AOP Orphan during the conduct of the studies; grants and personal fees from Novartis and personal fees from PharmaEssentia, MyeloPro Diagnostics and Research, Janssen-Cilag, Roche, and Celgene, outside of the work. $\mathrm{CK}, \mathrm{KK}$, and BG-I report that they were employees of AOP Orphan during the studies. JM reports grants from AOP Orphan during studies. RK reports personal fees from AOP Orphan Pharmaceuticals and PharmaEssentia, during the studies; personal fees from Qiagen and Novartis and stock ownership in MyeloPro Diagnostics and Research, outside of the work. HCH reports Data Monitoring Board honoraria from AOP Orphan during the studies; grants from Novartis outside of the work. J-JK reports grants and personal fees from AOP Orphan during the studies; grants and personal fees from Novartis and personal fees from Celgene, outside of the work. All other authors declare no competing interests.

\section{Data sharing}

Anonymised clinical study reports including all aggregated patient data, selected by-patient listings, study protocols, and the full statistical analysis plans will be shared indefinitely on completion of the

For more information on the European Medicines Agency see https://clinicaldata.ema. europa.eu mandatory disclosure process by the European Medicines Agency.

\section{Acknowledgments}

The study was funded by AOP Orphan Pharmaceuticals AG. Editorial assistance was provided by Victoria Empson, a medical writer funded by AOP Orphan Pharmaceuticals.

\section{References}

1 Griesshammer M, Gisslinger H, Mesa R. Current and future treatment options for polycythemia vera. Ann Hematol 2015; 94: 901-10.

2 Spivak JL. Polycythemia Vera. Curr Treat Options Oncol 2018; 19: 12.

3 Stein BL, Oh ST, Berenzon D, et al. Polycythemia Vera: an appraisal of the biology and management 10 years after the discovery of JAK2 V617F. J Clin Oncol 2015; 33: 3953-60.

4 Hasselbalch HC, Holmstrom MO. Perspectives on interferon-alpha in the treatment of polycythemia vera and related myeloproliferative neoplasms: minimal residual disease and cure? Semin Immunopathol 2019; 41: 5-19.

5 Kralovics R, Passamonti F, Buser AS, et al. A gain-of-function mutation of JAK2 in myeloproliferative disorders. $N$ Engl J Med 2005; 352: 1779-90.

6 Tefferi A, Barbui T. Essential Thrombocythemia and Polycythemia Vera: Focus on Clinical Practice. Mayo Clin Proc 2015; 90: 1283-93.

7 Vainchenker W, Kralovics R. Genetic basis and molecular pathophysiology of classical myeloproliferative neoplasms. Blood 2017; 129: 667-79.

8 Kiladjian JJ, Cassinat B, Turlure P, et al. High molecular response rate of polycythemia vera patients treated with pegylated interferon alpha-2a. Blood 2006; 108: 2037-40.

9 Kiladjian JJ, Cassinat B, Chevret S, et al. Pegylated interferon-alfa-2a induces complete hematologic and molecular responses with low toxicity in polycythemia vera. Blood 2008; 112: 3065-72.
10 King KY, Matatall KA, Shen CC, Goodell MA, Swierczek SI, Prchal JT. Comparative long-term effects of interferon alpha and hydroxyurea on human hematopoietic progenitor cells. Exp Hematol 2015; 43: 912-18.e2

11 Masarova L, Patel KP, Newberry KJ, et al. Pegylated interferon alfa-2a in patients with essential thrombocythaemia or polycythaemia vera: a post-hoc, median 83 month follow-up of an open-label, phase 2 trial. Lancet Haematol 2017; 4: e165-75.

12 Quintas-Cardama A, Kantarjian H, Manshouri T, et al. Pegylated interferon alfa-2a yields high rates of hematologic and molecular response in patients with advanced essential thrombocythemia and polycythemia vera. J Clin Oncol 2009; 27: 5418-24.

13 Silver RT, Kiladjian JJ, Hasselbalch HC. Interferon and the treatment of polycythemia vera, essential thrombocythemia and myelofibrosis. Expert Rev Hematol 2013; 6: 49-58.

14 Tashi T, Swierczek S, Kim SJ, et al. Pegylated interferon Alfa-2a and hydroxyurea in polycythemia vera and essential thrombocythemia: differential cellular and molecular responses. Leukemia 2018 32: 1830-33.

15 Them NC, Bagienski K, Berg T, et al. Molecular responses and chromosomal aberrations in patients with polycythemia vera treated with peg-proline-interferon alpha-2b. Am J Hematol 2015; 90: 288-94.

16 Verger E, Soret-Dulphy J, Maslah N, et al. Ropeginterferon alpha-2b targets JAK2V617F-positive polycythemia vera cells in vitro and in vivo. Blood Cancer J 2018; 8: 94.

17 Silver RT, Hasselbalch HC. Optimal therapy for polycythemia vera and essential thrombocythemia: Preferred use of interferon therapy based on phase 2 trials. Hematology 2016; 21: 387-91.

18 Quintas-Cardama A, Abdel-Wahab O, Manshouri T, et al Molecular analysis of patients with polycythemia vera or essential thrombocythemia receiving pegylated interferon alpha-2a. Blood 2013; 122: 893-901.

19 Gisslinger H, Zagrijtschuk O, Buxhofer-Ausch V, et al. Ropeginterferon alfa-2b, a novel IFNalpha-2b, induces high response rates with low toxicity in patients with polycythemia vera. Blood 2015; 126: 1762-69.

20 Jabbour E, Kantarjian H, Cortes J, et al. PEG-IFN-alpha-2b therapy in BCR-ABL-negative myeloproliferative disorders: final result of a phase 2 study. Cancer 2007; 110: 2012-18.

21 Silver RT. Long-term effects of the treatment of polycythemia vera with recombinant interferon-alpha. Cancer 2006; 107: 451-58.

22 Heis N, Rintelen C, Gisslinger B, Knobl P, Lechner K, Gisslinger H. The effect of interferon alpha on myeloproliferation and vascular complications in polycythemia vera. Eur J Haematol 1999; 62: 27-31.

23 Gisslinger H, Ludwig H, Linkesch W, Chott A, Fritz E, Radaszkiewicz T. Long-term interferon therapy for thrombocytosis in myeloproliferative diseases. Lancet 1989; 1: 634-37.

24 Alvarez-Larrán A, Pereira A, Cervantes F, et al. Assessment and prognostic value of the European LeukemiaNet criteria for clinicohematologic response, resistance, and intolerance to hydroxyurea in polycythemia vera. Blood 2012; 119: 1363-69.

25 Alvarez-Larrán A, Angona A, Ancochea A, at al. Masked polycythaemia vera: presenting features, response to treatment and clinical outcomes. Eur J Haematol 2016; 96: 83-89.

26 Vardiman JW, Thiele J, Arber DA, et al. The 2008 revision of the World Health Organization (WHO) classification of myeloid neoplasms and acute leukemia: rationale and important changes. Blood 2009; 114: 937-51.

27 Barosi G, Birgegard G, Finazzi G, et al. Response criteria for essential thrombocythemia and polycythemia vera: result of a European LeukemiaNet consensus conference. Blood 2009; 113: 4829-33.

28 Mascarenhas J, Kosiorek HE, Prchal JT, et al. Results of the Myeloproliferative Neoplasms-Research Consortium (MPN-RC) 112 randomized trial of pegylated interferon alfa-2a (PEG) versus hydroxyurea $(\mathrm{HU})$ therapy for the treatment of high risk polycythemia vera (PV) and high risk essential thrombocythemia (ET). Blood 2018; 132 (suppl 1): 577 (abstr)

29 Samuelsson J, Hasselbalch H, Bruserud O, et al. A phase II trial of pegylated interferon alpha-2b therapy for polycythemia vera and essential thrombocythemia: feasibility, clinical and biologic effects, and impact on quality of life. Cancer 2006; 106: 2397-405

30 Mullally A. Underlying mechanisms of the JAK2V617F mutation in the pathogenesis of myeloproliferative neoplasms. Pathologe 2016; 37 (suppl 2): 175-79. 
31 Kralovics R. Genetic complexity of myeloproliferative neoplasms. Leukemia 2008; 22: 1841-48.

32 Kiladjian JJ, Giraudier S, Cassinat B. Interferon-alpha for the therapy of myeloproliferative neoplasms: targeting the malignant clone. Leukemia 2016; 30: 776-81.

33 Gisslinger H, Klade C, Georgiev P, et al. Evidence for superior efficacy and disease modification after three years of prospective randomized controlled treatment of polycythemia vera patients with ropeginterferon alfa-2b vs. HU/BAT. Blood 2018; 132 (suppl 1): 579 (abstr)
34 Vannucchi AM, Antonioli E, Guglielmelli P, et al. Prospective identification of high-risk polycythemia vera patients based on JAK2(V617F) allele burden. Leukemia 2007; 21: 1952-59.

35 Cuthbert D, Stein BL. Therapy-associated leukemic transformation in myeloproliferative neoplasms-What do we know? Best Pract Res Clin Haematol 2019; 32: 65-73. 